\section{THU0569 A RANDOMISED, DOUBLE-BLIND, PARALLEL STUDY TO COMPARE RATES OF REMISSION (INACTIVE DISEASE) IN PATIENTS WITH JIA ON MTX TREATMENT ALONE VERSUS A COMBINATION OF MTX AND ETANERCEPT}

E. Alexeeva ${ }^{1,2}$, T. Dvoryakovskaya $^{1,2}$, R. Denisova ${ }^{1}$, I. Nikishina ${ }^{3}$, E. Zholobova ${ }^{2}$, V. Malievskiy ${ }^{4}$, G. Santalova ${ }^{5}$, E. Stadler ${ }^{5}$, L. Balykova ${ }^{6}$, Y. Spivakovskiy ${ }^{7}$, I. Becker ${ }^{8}, \mathrm{G}$. Horneff ${ }^{9} .{ }^{1}$ National Medical Research Center of Children's Health; ${ }^{2}$ Federal State Autonomous Educational Institution of Higher Education I.M. Sechenov First Moscow State Medical University, ${ }^{3}$ V.A. Nasonova Research Institute of Rheumatology, Moscow, ${ }^{4}$ Federal State Educational Institution of Higher Education Bashkir, Ufa; ${ }^{5}$ State Samara Medical University, Samara; ${ }^{6}$ Medical Institute of National Research Ogarev Mordovia State University, Saransk, ${ }^{7}$ Saratov State Medical University n.a. V. I. Razumovsky, Saratov, Russian Federation; ${ }^{8}$ University of Cologne, Cologne; ${ }^{9}$ Asklepios Clinic, Sankt Augustin, Germany

Background: Remission is the major goal of treatment of juvenile idiopathic arthritis(JIA). Advances in treatment options allowed achievement of remission to come into reach. Timing for initiating intensive treatment in a treat to target approach to early reach remission is still a debate.

Objectives: Multi-centre, double-blind, randomised study in polyarticular JIA patients (pts) receiving either Etanercept+Methotrexate (cohort 1) or Placebo +Methotrexate (cohort 2) for 24 weeks followed by 24 week open label phase. Escape to open-label ETN and MTX for pts not achieving paedACR30 at week 12 or not achieving inactive disease at week 24 .

Results: 35 pts were randomised to cohort 1 and 33 to cohort 2. Baseline demographic and clinical characteristics were comparable between the groups except mean tender joint count, physician assessment of global disease activity and JADAS10 which all were higher in cohort 1. At week 12, 12 pts randomised to cohort 2 did not reach paedACR30. At week 24, further 10 pts of cohort 2 not reaching inactive disease escaped to ETA and MTX. 5 pts of cohort 1 and 4 pts of cohort 2 dropped out. At week 12, significantly more pts on ETA and MTX (33 $(94 \%) / 27(79 \%))$ than on PLC and MTX (17 (52\%)/15 (47\%) reached paedACR30/50 ( $<<0.001 / 0.01)$. At week 24 , inactive disease was reached by 10 pts on ETA and MTX vs. 6 on PLC and MTX. Inactive disease at week 48 was achieved by 5 pts of cohort 2 on MTX alone while 22 patients (67\%) escaped to open label ETA and MTX. $15(43 \%)$ pts of cohort 1 and $16(49 \%)$ of cohort 2 reached inactive disease at week 48. At week 48, paedACR30/50/70/90 was reached by $100 \% / 97 \% / 97 \% / 77 \%$ in cohort 1 compared to $(97 \% / 93 \% / 93 \% / 73 \%)$ in cohort 2. The number of visits with inactive disease in cohort $1(87(24 \%))$ was comparable to that of cohort $2(79(21 \%))$ while the number of visits with no active joint was significantly higher in cohort $1(178(48 \%)$ vs. $146(39 \%)$; OR $1.45(1.08-$ 1.94); $p=0.012) .44(74 / 100 y)$ adverse events (AE) were noted leading to discontinuation in 6 patients. 3 serious AE occurred, two first uveitis events and one diagnosis of dermatomyositis, all in the placebo group. No new safety signal arised from our observation.

Conclusions: Early combination of ETA and MTX proved to be highly effective with a high rate of patients reaching high paediatric ACR response and $50 \%$ reaching inactive disease. Pre-defined targets to treat to, paediatric ACR30 at week12, defining minimal response or inactive disease at week 24/48 were more often reached upon ETA and MTX than with MTX alone. Compared to immediate intense antirheumatic combination treatment with ETA and MTX, a comparable rate of patients on targeted therapy reached the final endpoint of inactive disease at week 48 but numbers of visits with active arthritis were higher in patients receiving delayed combination therapy.

\section{REFERENCE:}

[1] Wallace CA, et al. Arthritis Care Res 2011;63:929-36.

Acknowledgements: The study was sponsored by an unrestricted grant by Pfizer, Russia

Disclosure of Interest: None declared

DOI: 10.1136/annrheumdis-2018-eular.2843

\section{THU0570 \\ LONG-TERM EFFICACY AND SAFETY OF CANAKINUMAB IN PATIENTS WITH COLCHICINE- RESISTANT FMF (CRFMF), TRAPS AND HIDS/MKD: RESULTS FROM THE PIVOTAL PHASE 3 CLUSTER TRIAL}

F. De Benedetti ${ }^{1}$, J. Frenkel ${ }^{2}$, A. Simon ${ }^{3}$, J. Anton ${ }^{4}$, H. Lachmann ${ }^{5}$, M. Gattorno ${ }^{6}$, S. Ozen ${ }^{7}$, I. Kone-Paut ${ }^{8}$, E. Ben-Chetrit ${ }^{9}$, M.B. Wozniak ${ }^{10}$, J.G. Wang ${ }^{11}$,

E. Vritzali ${ }^{11}{ }^{1} .{ }^{1}$ IRCCS Ospedale Bambino Gesú, Rome, Italy, ${ }^{2}$ University Medical Center Utrecht, Utrecht, ${ }^{3}$ Radboud University Medical Centre, Nijmegen, Netherlands: ${ }^{4}$ Hospital Sant Joan de Déu, Barcelona, Spain; ${ }^{5}$ UK National Amyloidosis Centre, University College London Medical School, London, UK; ${ }^{6}$ Pediatric Rheumatology, G. Gaslini Institute, Genoa, Italy, ${ }^{7}$ Hacettepe University Children's Hospital, Ankara, Turkey, ${ }^{8}$ Hôpital Kremlin Bicetre, University of Paris SUD, Paris, France; ${ }^{9}$ Hadassah - Hebrew University Medical Center, Jerusalem, Israel; ${ }^{10}$ Novartis Ireland Ltd, Dublin, Ireland; ${ }^{11}$ Novartis Pharma AG, Basel, Switzerland

Background: Canakinumab (CAN), a selective, human anti-interleukin (IL)-1 $1 \beta$ $\mathrm{mAb}$, has demonstrated efficacy and safety in patients (pts) with colchicine-resistant familial Mediterranean fever (crFMF), TNF receptor-associated periodic syndrome (TRAPS), and hyper-IgD syndrome (HIDS)/mevalonate kinase deficiency (MKD) in the epoch 2 and 3 (E2 and E3) of the CLUSTER study (NCT02059291). ${ }^{1}$

Objectives: To evaluate the long-term maintenance of optimal control of disease activity (median of no or 1 flare, and no uptitration) and safety on every 4 weeks $(\mathrm{q} 4 \mathrm{w})$ and every 8 weeks (q8w) dosing regimens of CAN in pts with crFMF, TRAPS or HIDS/MKD from the epoch 4 (E4; 112 weeks) of the CLUSTER study. Methods: The study comprised 4 epochs (E1-E4). Study design for E2 and E3 have been reported earlier. ${ }^{1}$ After lead-in E1, in E2, a 16 wk randomised, doubleblind, placebo (PBO)-controlled epoch, efficacy of CAN 150/300 mg q4w to induce complete response (absence of flares) was assessed. E3 (24 wks) evaluated whether responders to CAN 150/300 mg q4w in E2 could maintain clinical efficacy on 150/300 mg q8w or PBO. In E4, a 72 wk, open-label epoch, the long term maintenance of efficacy and safety of CAN $150 / 300 \mathrm{mg} \mathrm{q4w}$ or q8w in pts with crFMF, TRAPS or HIDS/MKD was evaluated. Pts who did not maintain clinical response on q8w could be uptitrated to $150 / 300 \mathrm{mg} \mathrm{q4w}$. Safety assessments included adverse events (AEs) and serious AEs.

Results: At the end of E4 (Wk 112), a substantial proportion of pts maintained optimal control of disease activity following treatment with 150/300 mg q4w or $q 8 w$ in all 3 cohorts (figure 1). HIDS/MKD pts more often required uptitration to $300 \mathrm{mg}$ q4w. Majority of pts in all 3 cohorts had 1 or no new flare (crFMF: $96.6 \%$, TRAPS: $94.3 \%$, HIDS/MKD: $83.3 \%$ ) and physician global assessment $<2$ (no or minimal disease activity). In all 3 cohorts, the median SAA levels decreased rapidly from baseline and remained suppressed through E4 (crFMF: 618 to $21 \mathrm{mg} / \mathrm{L}$, TRAPS: 243 to $12 \mathrm{mg} / \mathrm{L}$ and HIDS/MKD: 2061 to $16 \mathrm{mg} / \mathrm{L})$. No new safety findings were reported in CAN-treated pts through E4.

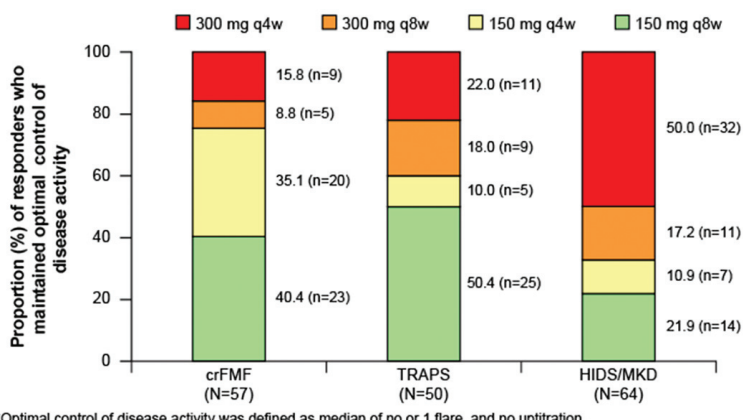

Abstract THU0570 - Figure 1 Proportion. of responders who maintained optimal control of disease activity ${ }^{*}$ at the end of epoch 4 (Week 112; following treatment with canakinumab $150 / 300 \mathrm{mg} \mathrm{q} 4 \mathrm{w}$ or $\mathrm{q} 8 \mathrm{w}$ ) 